\title{
Neonatal jaundice and stool production in breast- or formula-fed term infants
}

\author{
Hannah D. Buiter • Sebastiaan S. P. Dijkstra • \\ Rob F. M. Oude Elferink • Peter Bijster • \\ Henk A. Woltil • Henkjan J. Verkade
}

Received: 2 February 2007 / Accepted: 24 May 2007 / Published online: 10 July 2007

(C) Springer-Verlag 2007

\begin{abstract}
It has remained unclear whether the amount of fecal fat excreted in the stool and stool production influences the severity of neonatal jaundice. We determined the relationship between stool production, fecal fat excretion and jaundice in healthy breast-fed (BF) or formula-fed (FF) (near-)term neonates. From postnatal day 1-4, we quantitatively collected stools from $27 \mathrm{FF}$ and $33 \mathrm{BF}$ infants in daily fractions. Stool production and fecal fat contents were related to unconjugated bilirubin (UCB) levels, as determined by transcutaneous bilirubinometry $(\mathrm{TcB})$. Bilirubin concentrations and stool production did not differ between $\mathrm{FF}$ and $\mathrm{BF}$ neonates during the study period. Neonatal bilirubin levels were not inversely correlated with stool production. FF and BF infants had similar fecal fat excretion rates. The stool production of FF infants was profoundly lower in the present study than in a 1985 study by De Carvalho et al. [J Pediatr (1985) 107:786-790]. We conclude that
\end{abstract}

This study was supported by an unrestricted grant from Nutricia (Zoetermeer, The Netherlands).

Henkjan Verkade is a Research Fellow of the Royal Academy of Arts and Sciences.

H. D. Buiter · S. S. P. Dijkstra $\cdot$ H. J. Verkade

University Medical Center Groningen,

Groningen, The Netherlands

R. F. M. O. Elferink • P. Bijster $\cdot$ H. A. Woltil

Martini Hospital,

Groningen, The Netherlands

H. J. Verkade $(\square)$

Pediatric Gastroenterology/Hepatology,

Department Pediatrics, University Medical Center Groningen,

P.O. Box 30.001, 9700 RB

Groningen, The Netherlands

e-mail: h.j.verkade@bkk.umcg.nl increased jaundice during the first postnatal days in healthy term neonates can no longer be attributed to breast-feeding and speculate that improved absorbability of formulas since 1985 has contributed to similar fat excretion and stool production in FF and BF neonates in 2007.

Keywords Bilirubinometry - Enterohepatic circulation . Fat absorption · Newborns · Unconjugated bilirubin
Abbreviations
BF breast-fed
FF formula-fed
TcB transcutaneous bilirubinometry
UCB unconjugated bilirubin

\section{Introduction}

Neonatal jaundice is characterized by the accumulation of unconjugated bilirubin (UCB). UCB mainly originates from heme degradation in the reticulo-endothelial system and is transported to the liver for conjugation and subsequent biliary secretion. In neonates, hepatic conjugation activity is still low, whereas bilirubin production is transiently increased.

Breast-feeding has been associated on the one hand with an exaggeration of neonatal jaundice during the first postnatal days and, on the other hand, with a prolongation of the visible jaundice beyond the first 2 weeks of life ("breast-milk jaundice") $[10,12,19,24,32]$. The pathophysiology of increased neonatal jaundice in the first postnatal days in breast-fed (BF) infants has been a matter of debate. Weight loss in breast-fed infants is frequently 
higher than in formula-fed (FF) infants [19]. However, the difference in weight loss does not completely correspond to the higher incidence of jaundice during breast feeding $[1,4,6]$. Gourley and Arend suggested that the presence of $\beta$-glucuronidase in human breast milk - but not in formula milk - enhances the enterohepatic circulation of bilirubin [13], but subsequent studies failed to support this hypothesis $[2,17]$. Increased bilirubin absorption, rather than higher bilirubin production, has been suggested as an alternative mechanism underlying the exaggeration of breast milk jaundice $[2,3,11,16]$.

UCB can spontaneously diffuse through lipid bilayers and hepatocyt membranes [35]. Lester and Schmid and, more recently, Kotal et al. demonstrated that UCB can enter the intestinal lumen via biliary secretion and via transepithelial transport in Gunn rats and Crigler Najjar patients, both known to have persistent unconjugated hyperbilirubinemia [20-22]. We recently hypothesized that the neonatal disposal of UCB from the body is related to the amount of fat excreted via the feces [33]. According to this hypothesis, UCB associates with unabsorbed dietary fat in the intestinal lumen, which prevents its reabsorption (intestinal capture). The highly efficient fat absorption in BF infants compared with FF infants [34] may limit the disposal of UCB in the former and thus contribute to exaggerate neonatal hyperbilirubinemia. Subsequent studies in Gunn rats with spontaneous unconjugated hyperbilirubinemia supported the hypothesis. The stimulation of fecal fat excretion by treating Gunn rats with the lipase inhibitor orlistat increased fecal UCB excretion and decreased plasma UCB concentrations $[15,26]$.

No information is available on the relationship between neonatal jaundice and fecal fat excretion during the first postnatal days in infants. De Carvalho et al. demonstrated that in comparison to FF infants, $\mathrm{BF}$ infants had a lower stool production and fecal bilirubin output and higher serum bilirubin levels during the first 3 days of life [7]. Similarly, Gourley et al. reported a negative relationship between stool production and bilirubin levels in healthy term infants during the first 3 weeks of life [14]. Stool fat contents, however, were not quantified in these studies. Thus, these researchers were unable to infer whether the observed differences were actually due to lower fecal fat loss in BF infants.

The aim of the present prospective study was to determine the relationship between bilirubin concentrations, stool production and fecal fat excretion during the first postnatal days in healthy term $\mathrm{BF}$ and FF neonates. According to our hypothesis [33], lower fecal fat excretion rates would result in less fecal excretion of bilirubin and therefor higher levels of transcutaneously measured bilirubin. To allow comparison with previous data, the present study design closely mimicked that of De Carvalho et al. [7].

\section{Subjects and methods}

\section{Subjects}

The study protocol, based on informed parental consent, was approved by the Medical Ethics Committee of the Martini Hospital, The Netherlands. The BF group was breast-fed at least $75 \%$ of the daily ingested volume distributed over six to eight feedings daily. Data from infants whose total daily intake on any one of the first 5 postnatal days comprised between 25 and $75 \%$ formula milk were not included in the study. The fat composition of the formula was (per $100 \mathrm{~g}$ fatty acids) $38.3 \mathrm{~g}$ saturated fatty acids, $49.3 \mathrm{~g}$ monosaturated fatty acids and $12.4 \mathrm{~g}$ polyunsaturated fatty acids. The formula contained $20 \mathrm{~g}$ palmitic acid (C 16:0) per $100 \mathrm{~g}$ fatty acids.

\section{Study design}

Upon receiving parental consent, infants were enrolled immediately after birth. Inclusion criteria were gestational age of at least 36 weeks, Apgar score at $5 \mathrm{~min}$ of at least 7, uncomplicated delivery, either spontaneously or via cesarean section, and an expected stay in the hospital of at least 3 days. Neonates with meconium-stained amniotic fluid, polycythemia (defined as hematocrit above 0.60 ), positive Coombs-test, born by instrumental delivery, any apparent illness (including symptoms possibly due to infection) or use of medication were excluded. Maternal characteristics (parity, pre-existent morbidity), infant characteristics (Apgar score, sex, race, birth weight) were obtained and weight was recorded daily.

\section{Methods}

The extent of jaundice was measured daily at the same time in the morning using transcutaneous bilirubinometry $(\mathrm{TcB}$; BiliCheck, SpectRx, Norgross, Ga.). Each measurement consisted of placing the BiliCheck on the forehead for three times, after which the device calculated the average. The results were related to the post-partum age in hours at the time of measurement. During the study, transcutaneous bilirubin values were not used for clinical patient care.

From 48 of the 60 included patients $(27 \mathrm{BF}, 21 \mathrm{FF})$ all stools were quantitatively collected from birth until discharge using inlayer sheets in diapers. During the study period, fat-containing cream or lotion was avoided at the diaper area. Soiled inlayer sheets were pooled per day and frozen at $-20^{\circ} \mathrm{C}$ until analysis. Stool samples were treated according to the extraction procedure described by Jakobs et al. [18] with minor modifications. The total weight of the stools from 1 day was calculated by subtracting the weight 
of that day's inlayer sheets. The whole stool including the inlayer sheets per day was analyzed by mixing the stool with (per gram of stool) with $5 \mathrm{ml}$ of water, $1 \mathrm{ml}$ of $\mathrm{HCl}$ $(37 \%)$ and $12 \mathrm{ml}$ of ethanol $(96 \%)$ in a 1-1 plastic container. The mixture was vigorously shaken for $8 \mathrm{~min}$ using a paint container shaker (Minimix Mk3; Merris Development Engineers, Maidenhead, Berkshire, UK). A 10 -g sample of the suspension was put into a Teflon tube after which $10 \mathrm{ml}$ petroleum ether was added. The tube was vigorously shaken for $10 \mathrm{~min}$, followed by centrifugation for $10 \mathrm{~min}$ at $800 \mathrm{~g}, 10^{\circ} \mathrm{C}$. A $5-\mathrm{ml}$ portion of the organic layer was transferred to a glass tube and evaporated for $30 \mathrm{~min}$ at $40^{\circ} \mathrm{C}$ under a stream of nitrogen. The dried lipid extracts were dissolved in $1.0 \mathrm{ml}$ of chloroform (gradient grade) and transferred to a transmission flow cell (path length: $0.025 \mathrm{~mm}$ ) with sodium chloride crystals.

Spectra were measured in the mid-infrared region (4000$1300 \mathrm{~cm}^{-1}$ ). Ten scans were co-added at an optical resolution of $4 \mathrm{~cm}^{-1}$ using a Perkin-Elmer Spectrum 1000 Series Fourier Transform Infrared spectrometer (Perkin-Elmer, Norfolk Conn.). Chloroform was used for background subtraction. A primary standard mixture of stearic and palmitic acids (65:35) was used for the calibration curve in the range of $0-15 \mathrm{~g} \%$ using single linear regression analysis (Beer's Law). The primary standards were handled in the same way as the fecal samples. From the three characteristic peaks in the spectrum $\left(2927,2855\right.$ and $\left.1709 \mathrm{~cm}^{-1}\right)$, the heights at $2855 \mathrm{~cm}^{-1}$ was used for calculation.

\section{Statistics}

All results are presented as means \pm standard error of the mean. Differences between FF and BF neonates were evaluated by the Student's $t$-test or, in absence of a normal distribution, the Mann Whitney $U$-test. Correlations between parameters were calculated using Pearson rank correlation coefficient. The level of significance was set at $p<0.05$. The analysis was performed using SPSS ver. 10 for Windows software (SPSS, Chicago, Ill.).

\section{Results}

Table 1 shows the characteristics of the $\mathrm{BF}$ and $\mathrm{FF}$ infants studied. Thirty-three BF and 27 FF (Nutrilon Premium) infants were studied during the first postnatal days $(4 \pm 1$ days, mean \pm SEM). FF infants received at least $75 \%$ of the daily volume intake as formula-feeding. Initially, 64 infants had been included, but the results of four infants were not used for the analyses because of incomplete data $(n=2)$, discharge on the second postnatal day $(n=1)$ or severe hyperbilirubinemia requiring phototherapy on the second
Table 1 Characteristics of the breast-fed and formula-fed group of infants

\begin{tabular}{lll}
\hline & $\begin{array}{l}\text { Breast-fed } \\
\text { infants }\end{array}$ & $\begin{array}{l}\text { Formula-fed } \\
\text { infants }\end{array}$ \\
\hline$n$ & 33 & 27 \\
Birth weight (kg) & $3.3 \pm 0.1$ & $3.3 \pm 0.1$ \\
Gestational age (weeks) & $39.1 \pm 0.2$ & $38.6 \pm 0.3$ \\
Weight loss (\% of birth weight) & $7.6 \pm 0.4^{*}$ & $5.9 \pm 0.5^{*}$ \\
Parity (\% nulliparae) & $22(67 \%)$ & $6(22 \%)$ \\
Male (\% of all infants) & $16(48 \%)$ & $13(48 \%)$ \\
Caesarian section & $31(94 \%)$ & $21(78 \%)$ \\
(\% of all infants) & & \\
\hline
\end{tabular}

All values are means \pm standard error of the mean (SEM).

${ }^{*} p<0.05$. There were no significant differences between $\mathrm{BF}$ and $\mathrm{FF}$ infants in any of the other parameters.

postnatal day $(n=1)$. Of the remaining 60 infants (gestational age: $39.2 \pm 0.2$ weeks), one FF infant developed hyperbilirubinemia at day 4 and was treated with phototherapy. The data of this infant were included in the analysis until the start of phototherapy. The two groups did not differ significantly in birth weight, gestational age, male/female distribution or delivery via caesarean section (Table 1). The rate of cesarean section is high, attributable to the inclusion criterium of healthy (near-)term infants with an expected stay in the hospital of at least 3 days. The fraction of first-borns was higher in the BF group than in the FF group (67 vs. $23 \%$, respectively). BF infants lost significantly more weight than FF infants during the study period ( $p=0.013$; Table 1).

Figure 1 shows that the $\mathrm{TcB}$ values were very similar in the $\mathrm{BF}$ and $\mathrm{FF}$ group during the first 4 postnatal days. Maximum bilirubin levels during the study period were reached at the fourth day: $153 \pm 10$ and $146 \pm 10 \mu \mathrm{mol} / 1$ in $\mathrm{BF}$ and $\mathrm{FF}$ infants, respectively. Levels in the BF group seemed to level off at day 4 , in contrast to the FF group. No significant differences in $\mathrm{TcB}$ were found between $\mathrm{BF}$ and FF infants on any of the study days. Although BF infants lost more bodyweight, neonatal weight loss was not significantly related to $\mathrm{TcB}$ levels in either group (FF; $r^{2}=0.01, \mathrm{BF} ; r^{2}=0.05$ ).

Figure 2 shows that the stool production was similar between the BF and FF infants on any one of the days. Cumulative fecal weight in the first 4 postnatal days was $49 \pm 4 \mathrm{~g}$ in BF infants and $46 \pm 4 \mathrm{~g}$ in FF infants, a non-significant difference. Figure 3 shows the cumulative fecal fat excretion in the two groups. Between birth and postnatal day $4, \mathrm{BF}$ infants had a cumulative fecal fat excretion of $3.2 \pm 0.7 \mathrm{~g}$, which was similar to the $3.9 \pm 1.3 \mathrm{~g}$ excreted by FF infants. 


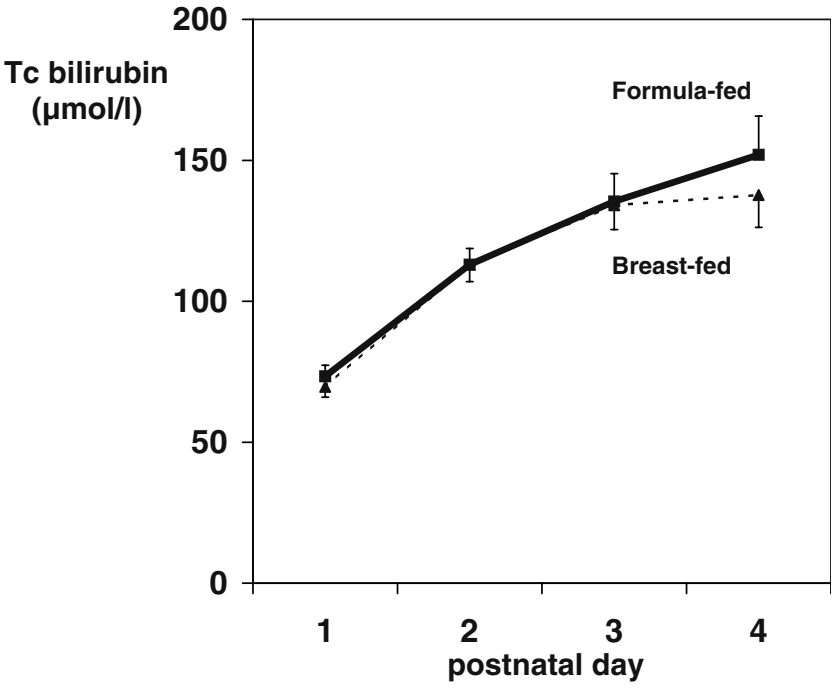

Fig. 1 Transcutaneous bilirubin levels measured in breast-fed (BF) and formula-fed (FF) infants during the first postnatal days. Bilirubin levels did not differ significantly between BF $(n=33)$ and FF $(n=27)$ in any of the first 4 days after birth. Values represent means \pm standard error of the mean (SEM). No significant differences were found. Tc Transcutaneous. [17.1 $\mu \mathrm{mol} / \mathrm{l}$ unconjugated bilirubin (UCB) equals $1 \mathrm{mg} / \mathrm{dl} \mathrm{UCB}]$

\section{Discussion}

We investigated the relationships between stool production, fecal fat excretion and jaundice in healthy BF and FF term neonates. In contrast to previous studies, we found that BF and FF infants had similar levels of jaundice and stool production during the first postnatal days.

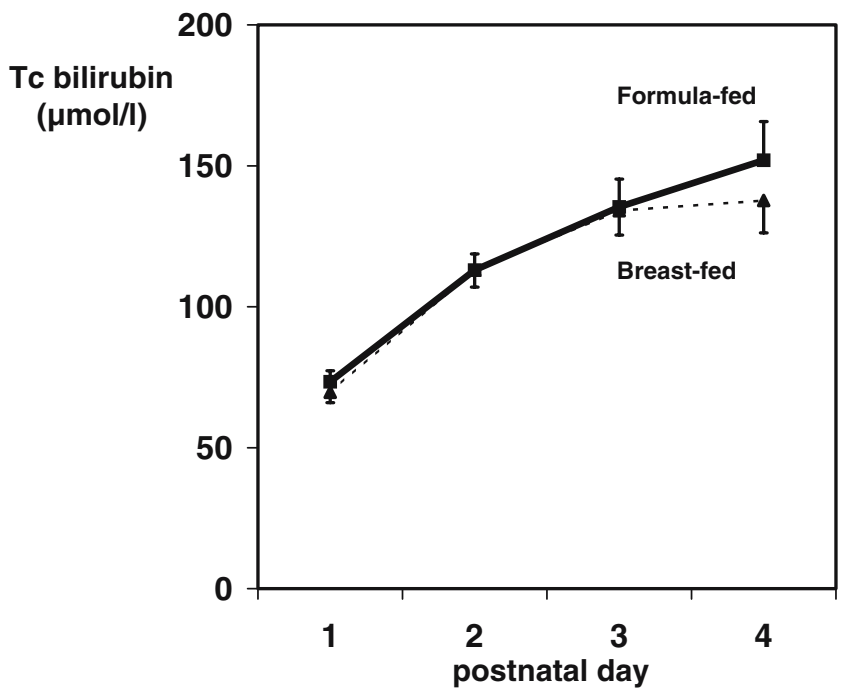

Fig. 2 Cumulative stool production in FF and BF infants during the first 4 postnatal days In the first 4 postnatal days the total amount of stool production did not significantly differ between $\mathrm{BF}(n=27)$ and FF $(n=21)$ infants. Values represent means \pm SEM. Differences between the two groups of infants were not statistically significant at any of the days studied

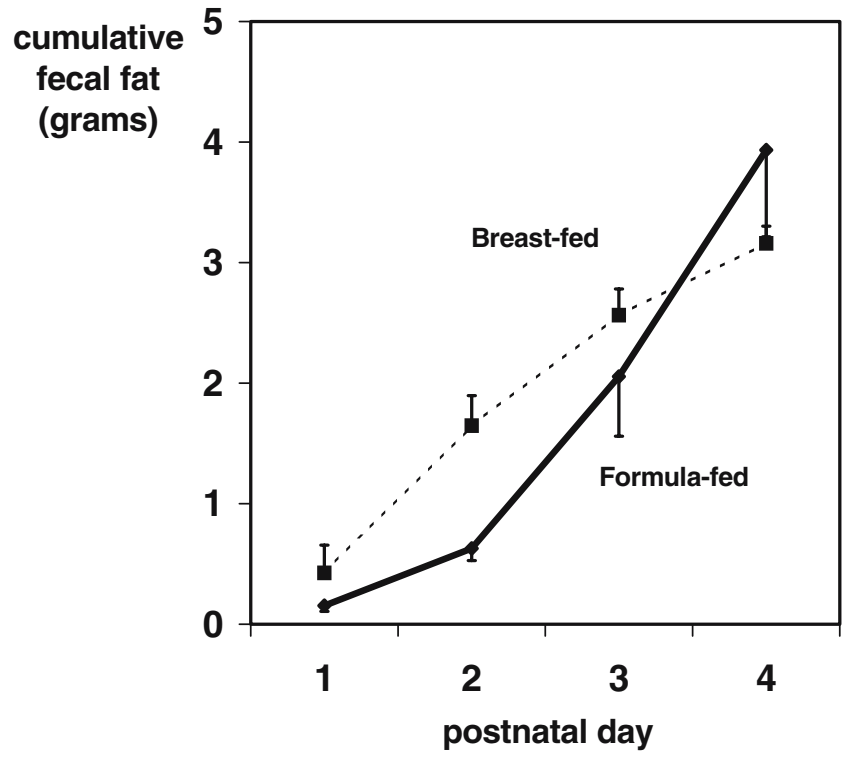

Fig. 3 Cumulative fecal fat excretion (in grams per day) in BF $(n=27)$ and FF infants $(n=21)$ Cumulative fecal fat excretion in FF infants was similar to that in BF infants during first 4 postnatal days. Values represent means \pm SEM. No significant differences were found

De Carvalho et al. reported a negative linear correlation between neonatal stool production and serum bilirubin concentrations [7]. In the present study, however, TcB levels were neither related to cumulative stool production (Fig. 4) nor to neonatal fecal fat excretion (Fig. 5). Many factors have been implied to influence the severity of neonatal jaundice, such as parity, weight loss, mode of delivery, gestational age, and maternal characteristics such as age and smoking. It needs to be realized, however, that the various studies on these factors are not conclusive and sometimes even contradictory $[1,6,9,23,25,30]$. In our

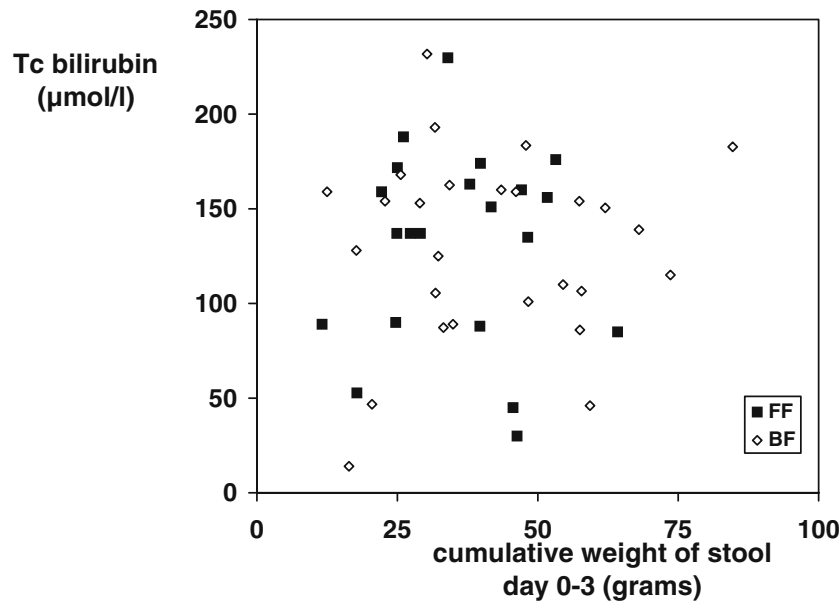

Fig. 4 The relationship between transcutaneous $(T c)$ bilirubin levels and cumulative stool production in the first postnatal days of $\mathrm{BF}$ and FF term neonates. TcB levels in both $\mathrm{BF}$ and FF infants $(n=48)$ at postnatal day 3 are not correlated with cumulative stool production between post-partum day 0 and day $3\left(R^{2}=0.0055\right.$ for $\mathrm{BF}, R^{2}=0.0004$ for FF infants) 


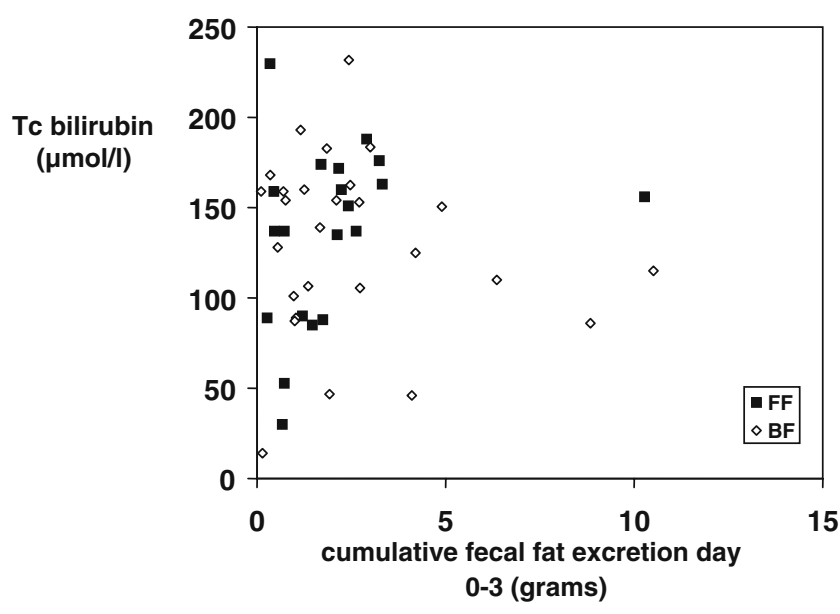

Fig. 5 The relationship between $\mathrm{TcB}$ levels and cumulative fecal fat excretion in the first postnatal days of $\mathrm{BF}$ and $\mathrm{FF}$ term neonates. TcB levels in both $\mathrm{BF}$ and $\mathrm{FF}$ infants $(n=48)$ at postnatal day 3 are not correlated with cumulative fecal fat excretion between day 0 and day 3 $\left(r^{2}=0.019\right.$ for BF, $r^{2}=0.065$ for $\mathrm{FF}$ infants $)$

study, neonatal weight loss and parity differed between $\mathrm{BF}$ and FF infants. We do not consider it likely, however, that this could account for the similar degree of neonatal jaundice in the two groups. A higher weight loss (possibly due to decreased nutritional intake) was found in the BF infants, and one could have expected higher bilirubin concentrations in BF infants, what we did not observe. We also did not consider it likely that the trancutaneous analysis of bilirubin levels is responsible for the lack of difference between $\mathrm{BF}$ and $\mathrm{FF}$ infants. In the range of bilirubin values reported in the present study, the TcB measurement is accurate, reproducible and comparable to the standard of care laboratory tests, [4, 5, 28, 29]. We considered the possibility that the relatively small number of infants could mask possible differences between BF and FF infants. However, De Carvalho et al. did find significant differences with $40 \%$ fewer infants. It therefore seems likely that the lack of difference in bilirubin levels between the two groups of infants in our study cannot be attributed to these various factors. The apparent discrepancy between the present observations and the data reported previously by De Carvalho et al. was further analyzed (Fig. 6). BF infants in De Carvalho et al.'s study and in the present study produced rather similar amounts of stool (estimated difference of approx. $20 \%$ based on figures; original data were not available; personal communication with $\mathrm{M}$. De Carvalho). FF infants in the present study, however, produced profoundly lower levels of stools compared to the FF infants reported by De Carvalho (cumulative stool weight: 36 vs. $80 \mathrm{~g}$ at day 3 , respectively). Since the results of BF infants were similar, we consider it unlikely that differences in the methodology of feces collection or weighing could account for the results of the FF infants, who were subjected to identical procedures. Rather, we

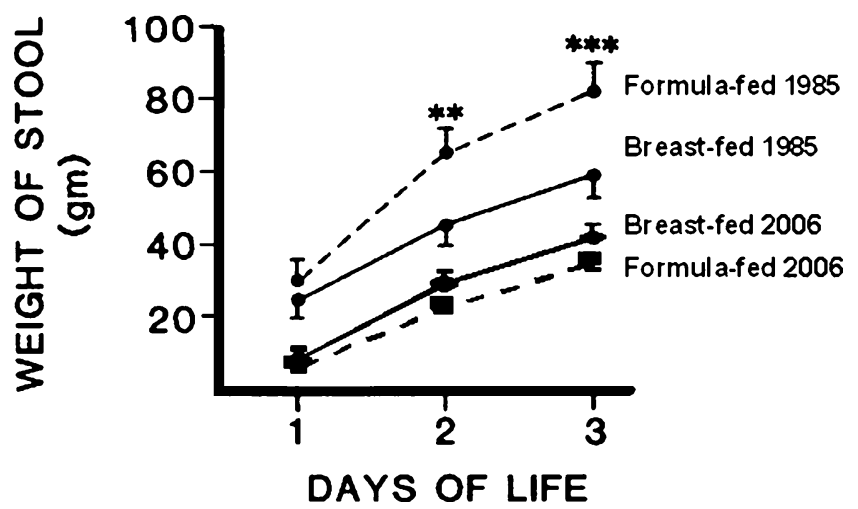

Fig. 6 Cumulative weight of stools in the first postnatal days in BF and FF infants: a comparison of data from De Carvalho et al.'s 1985 study [7] and the present study. FF infants in the present study produced less stool than the FF infants in De Carvalho et al.'s study. In contrast, BF infants produced similar amounts of stool as those studied in 1985

hypothesize that differences in the formulas used, and therefore differences in intestinal (fat-)absorbability, may play a role in the apparent discrepancy. Modernization of formula-feeding may have led to improved absorbability and correspondingly to lower fat malabsorption in FF infants. Indeed, fecal fat excretion in FF infants is approaching the levels of excretion in BF infants [27]. Upon requesting information concerning possible changes in the composition of infant nutrition with a major supplier in the Netherlands, Nutricia B.V., we learned that the contribution of medium-chain triglycerides was increased in 1991 by $43 \%$ (from 14.3 to $20.5 \mathrm{~g} / 100 \mathrm{~g}$ fatty acids). It is well known that medium-chain triglycerides have a higher absorbability than long(er) chain triglycerides. Detailed information on fat content and composition of the formula used in De Carvalho et al.'s study was not available.

Differences in neonatal bilirubin levels between $\mathrm{BF}$ and FF infants have been described frequently in the past. More recently, however, it has been questioned whether BF infants in general have higher bilirubin levels. Bertini et al. measured UCB of more than 2000 term infants in the first postnatal days and found that only a small subgroup of the BF infants had significant hyperbilirubinemia, possibly related to fasting [3]. Our data are in accordance with those of Bertini et al. and, together with results from other recent studies, indicate that the extent of neonatal jaundice in the first postnatal days is similar in FF and BF infants. The possible mechanism underlying this phenomenon is unclear.

The neonatal TcB levels of the present study are similar to previously reported average bilirubin levels in term infants in the first postnatal days [8]. Peak bilirubin levels are normally found on postnatal days 4 or 5 in term infants [8]. Our own data indeed indicate that the bilirubin concentrations level off at day 4 (Fig. 1). Due to the standard release of mothers and neonates from the hospital 
at day 3 or 4 , we could only measure TcB and collect feces until this time point.

In our study, the incidence of severe hyperbilirubinemia requiring phototherapy was $1.6 \%$, which is low compared to a known incidence of $5.1-10.5 \%[3,24,31]$. This is probably due to the exclusion of infants with a known high risk of hyperbilirubinemia from the study. Our study included a high number of infants born by caesarian section who are known to have a decreased risk of hyperbilirubinemia. De Carvalho et al. reported somewhat higher bilirubin levels in the first postnatal days, possibly due to a high number of infants with forceps delivery [7].

In summary, we originally hypothesized that the difference in the extent of neonatal jaundice between $\mathrm{FF}$ and $\mathrm{BF}$ infants was related to the efficacy of fat (mal)absorption. However, the similarity in neonatal jaundice (and stool production) observed in the present study provide an indication for a "change in paradigm" with respect to the effect of breast feeding on the exaggeration of neonatal jaundice during the first postnatal days. It is tempting to speculate that, in accordance with our hypothesis, the now similar fecal fat excretion rates in $\mathrm{BF}$ and $\mathrm{FF}$ infants has led to an equally high ability to capture UCB in the intestinal lumen and prevent its enterohepatic circulation.

\section{References}

1. Adams JA, Hey DJ, Hall RT (1985) Incidence of hyperbilirubinemia in breast- vs. formula-fed infants. Clin Pediatr 24(2):69-73

2. Alonso EM, Whitington PF, Whitington SH, Rivard WA, Given G (1991) Enterohepatic circulation of nonconjugated bilirubin in rats fed with human milk. J Pediatr 118(3):425-430

3. Bertini G, Dani C, Tronchin M, Rubaltelli FF (2001) Is breastfeeding really favoring early neonatal jaundice? Pediatrics 107(3):E41

4. Bertini G, Rubaltelli FF (2002) Non-invasive bilirubinometry in neonatal jaundice. Semin Neonatol 7(2):129-133

5. Bhutani VK, Gourley GR, Adler S, Kreamer B, Dalin C, Johnson LH (2000) Noninvasive measurement of total serum bilirubin in a multiracial predischarge newborn population to assess the risk of severe hyperbilirubinemia. Pediatrics 106(2):E17

6. Bracci R, Buonocore G, Garosi G, Bruchi S, Berni S (1989) Epidemiologic study of neonatal jaundice. A survey of contributing factors. Acta Paediatr Scand Suppl 360:87-92

7. De Carvalho M, Robertson S, Klaus M (1985) Fecal bilirubin excretion and serum bilirubin concentrations in breast-fed and bottle-fed infants. J Pediatr 107(5):786-790

8. Fanaroff M (2002) Neonatal jaundice and liver disease. In: Neonatal and perinatal medicine: diseases of the fetus and infant. Mosby, St. Louis, pp 1315-1317

9. Gale R, Seidman DS, Dollberg S, Stevenson DK (1990) Epidemiology of neonatal jaundice in the Jerusalem population. J Pediatr Gastroenterol Nutr 10(1):82-86

10. Gartner LM, Herschel M (2001) Jaundice and breastfeeding. Pediatr Clin North Am 48(2):389-399
11. Gartner LM, Lee KS, Moscioni AD (1983) Effect of milk feeding on intestinal bilirubin absorption in the rat. J Pediatr 103(3): 464-471

12. Gourley GR (2002) Breast-feeding, neonatal jaundice and kernicterus. Semin Neonatol 7(2):135-141

13. Gourley GR, Arend RA (1986) beta-Glucuronidase and hyperbilirubinaemia in breast-fed and formula-fed babies. Lancet 1 (8482):644-646

14. Gourley GR, Kreamer B, Arend R (1992) The effect of diet on feces and jaundice during the first 3 weeks of life. Gastroenterology 103(2):660-667

15. Hafkamp AM, Havinga R, Sinaasappel M, Verkade HJ (2005) Effective oral treatment of unconjugated hyperbilirubinemia in Gunn rats. Hepatology 41(3):526-534

16. Hintz SR, Gaylord TD, Oh W, Fanaroff AA, Mele L, Stevenson DK (2001) Serum bilirubin levels at 72 hours by selected characteristics in breastfed and formula-fed term infants delivered by cesarean section. Acta Paediatr 90(7):776-781

17. Ince Z, Coban A, Peker I, Can G (1995) Breast milk betaglucuronidase and prolonged jaundice in the neonate. Acta Paediatr 84(3):237-239

18. Jakobs BS, Volmer M, Swinkels DW, Hofs MT, Donkervoort S, Joosting MM, Wolthers BG, de Peinder P, Voorbij HA (2000) New method for faecal fat determination by mid-infrared spectroscopy, using a transmission cell: an improvement in standardization. Ann Clin Biochem 37(Pt 3):343-349

19. Johnson CA, Lieberman B, Hassanein RE (1985) The relationship of breast feeding to third-day bilirubin levels. J Fam Pract 20 (2):147-152

20. Kotal P, Van der Veere CN, Sinaasappel M, Elferink RO, Vitek L, Brodanova M, Jansen PL, Fevery J (1997) Intestinal excretion of unconjugated bilirubin in man and rats with inherited unconjugated hyperbilirubinemia. Pediatr Res 42(2):195-200

21. Lester R, Schmid R (1963) Intestinal absorption of bile pigments. I. The enterohepatic circulation of bilirubin in the rat. J Clin Invest 42:736-746

22. Lester R, Schmid R (1963) Intestinal absorption of bile pigments. II. Bilirubin absorption in man. N Engl J Med 269:178-182

23. Linn S, Schoenbaum SC, Monson RR, Rosner B, Stubblefield PG, Ryan KJ (1985) Epidemiology of neonatal hyperbilirubinemia. Pediatrics 75(4):770-774

24. Maisels MJ, Gifford K (1986) Normal serum bilirubin levels in the newborn and the effect of breast-feeding. Pediatrics 78(5):837843

25. Maisels MJ, Gifford K, Antle CE, Leib GR (1988) Jaundice in the healthy newborn infant: a new approach to an old problem. Pediatrics 81(4):505-511

26. Nishioka T, Hafkamp AM, Havinga R, vn Lierop PP, Velvis H, Verkade HJ (2003) Orlistat treatment increases fecal bilirubin excretion and decreases plasma bilirubin concentrations in hyperbilirubinemic Gunn rats. J Pediatr 143(3):327-334

27. Rings EH, Minich DM, Vonk RJ, Stellaard F, Fetter WP, Verkade HJ (2002) Functional development of fat absorption in term and preterm neonates strongly correlates with ability to absorb long-chain fatty acids from intestinal lumen. Pediatr Res 51(1):57-63

28. Robertson A, Kazmierczak S, Vos P (2002) Improved transcutaneous bilirubinometry: comparison of SpectR(X) BiliCheck and Minolta Jaundice Meter JM-102 for estimating total serum bilirubin in a normal newborn population. J Perinatol 22(1): $12-14$

29. Rubaltelli FF, Gourley GR, Loskamp N, Modi N, Roth-Kleiner M, Sender A, Vert P (2001) Transcutaneous bilirubin measurement: a multicenter evaluation of a new device. Pediatrics 107(6): $1264-1271$ 
30. Saigal S, Lunyk O, Bennett KJ, Patterson MC (1982) Serum bilirubin levels in breast- and formula-fed infants in the first 5 days of life. Can Med Assoc J 127(10):985-989

31. Sarici SU, Serdar MA, Korkmaz A, Erdem G, Oran O, Tekinalp G, Yurdakok M, Yigit S (2004) Incidence, course, and prediction of hyperbilirubinemia in near-term and term newborns. Pediatrics 113(4):775-780

32. Schneider AP (1986) Breast milk jaundice in the newborn. A real entity. JAMA 255(23):3270-3274
33. Verkade HJ (2002) A novel hypothesis on the pathophysiology of neonatal jaundice. J Pediatr 141(4):594-595

34. Verkade HJ, Hoving EB, Muskiet FA, Martini IA, Jansen G, Okken A, Vonk RJ, Bijleveld CM (1991) Fat absorption in neonates: comparison of long-chain-fatty-acid and triglyceride compositions of formula, feces, and blood. Am J Clin Nutr 53(3):643-651

35. Zucker SD, Goessling W, Hoppin AG (1999) Unconjugated bilirubin exhibits spontaneous diffusion through model lipid bilayers and native hepatocyte membranes. J Biol Chem 274(16):10852-10862 